International Journal of Pure and Applied Mathematics

Volume 92 No. 4 2014, 587-590

ISSN: 1311-8080 (printed version); ISSN: 1314-3395 (on-line version)

url: http://www.ijpam.eu

doi: http://dx.doi.org/10.12732/ijpam.v92i4.13

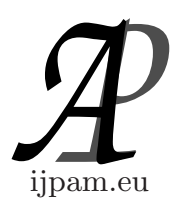

\title{
REMARK ON SUBOBJECTS IN A TOPOS
}

\author{
Tao $\mathrm{Lu}$ \\ ${ }^{1}$ School of Mathematics Science \\ HuaiBei Normal University \\ HuaiBei, AnHui, 235000, P.R. CHINA
}

Abstract: In this paper, we study the partially subobjects in a topos, and the equivalence between $S+T$ and $S \cup T$ is obtained.

AMS Subject Classification: 06B30, 18B25

Key Words: coproduct, topos, subobject

\section{Introduction and Preliminaries}

Recall a topos $\mathcal{E}$ is a category which has finite limits and every object of $\mathcal{E}$ has a power object. For a fixed object $A$ of category $\mathcal{E}$, the power object of $A$ is an object $P A$ which represents $\operatorname{Sub}(-\times A)$, so that $\operatorname{Hom}_{\mathcal{E}}(-, P A) \simeq \operatorname{Sub}(-\times A)$ naturally. It says precisely that for any arrow $B^{\prime} \stackrel{f}{\longrightarrow} B$, the following diagram commutes, where $\varphi$ is the natural isomorphism.

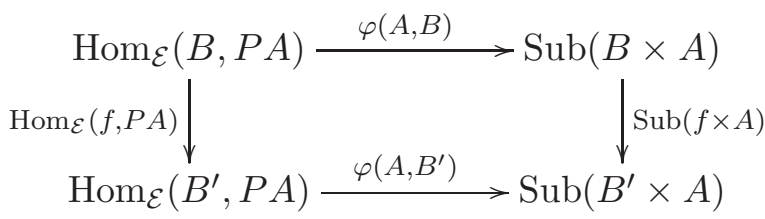

Figure 1

As a matter of fact, the category of sheaves of sets on a topological space is a topos. In particular, the category of sets is a topos. For details of the treatment of toposes and sheaves please see Johnstone [1], Mac and Moerdijk [2], Joyal

Received: February 8, 2014

(c) 2014 Academic Publications, Ltd. url: www.acadpubl.eu 
and Tierney [3], Johnstone and Joyal [4]. For a general background on category theory please refer to [5], [6]

In [2], Lattice and Heyting Algebra objects in a topos are well defined. In [xxx] the Partially ordered objects are well defined too. In this paper, we want to give some properties about subobjects of partially ordered objects.

More details about lattice and locale please see [7], [8], [9], [10].

\section{Main Results}

Throughout this paper, we work with a fixed topos $\mathscr{E}$. All objects mentioned belong to the topos $\mathscr{E}$.

In a topos $\mathscr{E}$, given two subobjects $S \longmapsto A$ and $T \longmapsto A$ we can define the greatest lower bound in the partially ordered set $\operatorname{Sub} A$ of subjects of $A$ by taking the following pullback as following:

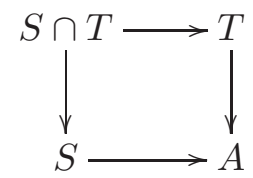

And then we denote $S+T$ as the coproduct of the subobjects $S \longmapsto A$ and $T \longmapsto A, M$ is the image of $S+T$, as shown in the following diagram:

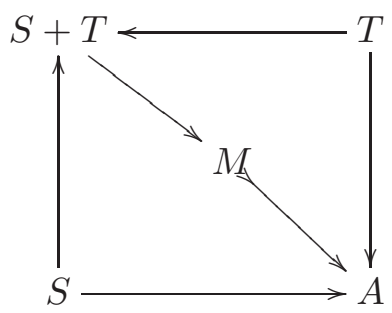

then, $M \longmapsto A$ belongs to $\operatorname{Sub} A$ which contains $S$ and $T$ and so $M$ is the least upper bound of $S$ and $T$ in $\operatorname{Sub} A$.

Lemma 1. [1] In a topos, every morphism $f$ has an image $m$ and factors as $f=$ me, with e epi.

Proposition 2. For any partially ordered object $B$ in a topos $\mathscr{E}$, the inclusion $i: S u b A \rightarrow \mathscr{E} / B$ has a left adjoint $k$ which sends each $f: A \rightarrow B$ to its image. 
Proof. For each object $f: A \rightarrow B$ of the slice category $\mathscr{E} / B$ take $k f$ to be the image $m: M \longmapsto B$,regarded as a subobject of $B$. And if $f$ factors through a monic $h: C^{\prime} \rightarrow B$, so does $m$ by Lemma 1 . This shows that $\operatorname{Hom}_{\mathscr{E} / B}(f, h) \cong \operatorname{Hom}_{S u b(B)}(m, h)$. Hence $k$ defined by $k f=m$ is the required left adjoint to the inclusion $i$.

Theorem 3. Let $\mathscr{E}$ be a topos and $B$ a partially ordered object. If $S$ and $T$ are disjoint subobjects of $B$, then the join $S \cup T$ in $B$ is isomorphic to the coproduct $S+T$.

Proof. Let $h: S \longmapsto B$ and $k: T \longmapsto B$ be the conclusions of the given two subobjects. Their coproduct $S+T$ in $\mathscr{E}$ is also their coproduct in $\mathscr{E} / B$. The pullback functor $k^{*}: \mathscr{E} / B \rightarrow \mathscr{E} / T$ is a left adjoint, hence preserves the coproduct $\langle h, k\rangle: S+T \rightarrow B$ in $\mathscr{E} / B$ as the second square shows in the following diagram.

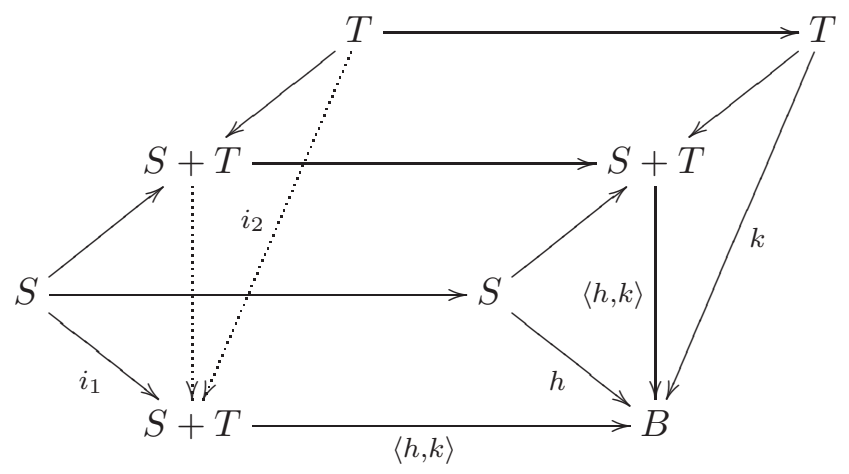

Now the pullback $k^{*} k$ is the idendity, hence the pullback of $S+T \rightarrow B$ along $k$ is the idendity $T \rightarrow T$ as the third square shows in the above diagram.

Similarily, this means the pullback along $\langle h, k\rangle$ turns $k$ into the inclusion $i_{2}: T \rightarrow S+T$ and turns $h$ into $i_{1}: S \rightarrow S+T$ as the first and the second square show in the above diagram.

Since the pullback functor $\langle h, k\rangle^{*}$ preserves coproducts, the pullback of $S+T \rightarrow B$ along itself is the idendity as the second square shows. This in turn shows $S+T \rightarrow B$ is mono. Hence, $S+T$ is a subobject of $B$. So, by the definition of the join $S \cup T$, the join is $S+T$.

\section{Acknowledgments}

The author would like to thank the referee for his helpful suggestions. 
The work is supported by the National Natural Science Foundation of (Grants No.11171156) and the Doctor Foundation(Grant No.600483) and Science Foundation of AnHui Provience (Grant No.KJ2012Z358).

\section{References}

[1] P.T. Johnstone, Sketches of an Elephant: A Topos Theory Compendium, The Clarendon Press, Oxford University Press, Oxford(2002).

[2] L.C. MAC, MOERDIJK I, Sheaves in Geometry and Logic, SpringerVerlag, New York(1994).

[3] A. JOYAL, M. TIERNEY, An extension of the Galois theory of Grothendieck, Mem. Amer. Math. Soc., 51(309)(1984), 71.

[4] P.T. Johnstone, JOYAL A, Continuous categories and exponentiable toposes, J. Pure Appl. Algebra, 25(3)(1982), 255-296.

[5] He Wei, Category Theory, Beijing: Science Press(2006). (in Chinese)

[6] L.C. MAC, Categories for the Working Mathematician, Springer-Verlag, New York-Berlin(1971).

[7] J.R. ISBELL, Atomless parts of spaces, Math. Scand., 31(1972), 5-32.

[8] J.R. ISBELL, First steps in descriptive theory of locales, Trans. Amer. Math. Soc., 327(1)(1991), 353-371.

[9] He Wei, LIU Yingming, Steenrod's theorem for locales, Math. Proc. Cambridge Philos. Soc., 124(2)(1998), 305-307.

[10] P.T. Johnstone. Stone Spaces, Cambridge University Press, Cambridge, 1982.

[11] Lu Tao, He Wei, Wang XiJuan, Meets of spatial sublocales, Bulletin of the Belgian Mathematical Society. Simon Stevin , 17(2010), 243-250.

[12] Lu Tao, The factorization of monotone morphism in a topos, International journal of pure and applied mathematics, 63(2)(2010), 159-168. 